\title{
A D-N Alternating Algorithm for Solving 3D Exterior Helmholtz Problems
}

\author{
Qing Chen, ${ }^{1}$ Baoqing Liu, ${ }^{2}$ and Qikui Du ${ }^{1}$ \\ ${ }^{1}$ School of Mathematical Sciences, Jiangsu Provincial Key Laboratory for NSLSCS, Nanjing Normal University, Nanjing 210023, China \\ ${ }^{2}$ School of Applied Mathematics, Jiangsu Provincial Key Laboratory for NSLSCS, Nanjing University of Finance and Economics, \\ Nanjing 210023, China \\ Correspondence should be addressed to Qikui Du; duqikui@njnu.edu.cn
}

Received 6 May 2014; Revised 3 July 2014; Accepted 3 July 2014; Published 20 July 2014

Academic Editor: Xiao-Qiao He

Copyright (C) 2014 Qing Chen et al. This is an open access article distributed under the Creative Commons Attribution License, which permits unrestricted use, distribution, and reproduction in any medium, provided the original work is properly cited.

\begin{abstract}
The nonoverlapping domain decomposition method, which is based on the natural boundary reduction, is applied to solve the exterior Helmholtz problem over a three-dimensional domain. The basic idea is to introduce a spherical artificial boundary; the original unbounded domain is changed into a bounded subdomain and a typical unbounded region; then, a Dirichlet-Nuemann (D-N) alternating method is presented; the finite element method and natural boundary element methods are alternately applied to solve the problems in the bounded subdomain and the typical unbounded subdomain. The convergence of the D-N alternating algorithm and its discretization are studied. Some numerical experiments are presented to show the performance of this method.
\end{abstract}

\section{Introduction}

Many scientific and engineering problems can be reduced to exterior boundary value problems of partial differential equations. Although the numerical methods for solving boundary value problems, such as the finite element method and the finite difference method, are very effective on a bounded domain, yet we often find it difficult to be applied to unbounded problem directly. To solve such problems in infinite region numerically, there are a variety of numerical methods, cf. [1-3] and references therein for more details.

There have been studied for Dirichlet-Nuemann alternating algorithm, or D-N alternating algorithm on $2 \mathrm{D}$ unbounded domain problems extensively, such as Possion equation [4] and Helmholtz equation [5]. Wu and Yu [6] studied the natural integral equations of 3D Helmholtz problems. Jia et al. [7] investigated the coupled natural boundary element-finite element method for solving 3D exterior Helmholtz problem.

In this paper, based on the natural boundary reduction, a $\mathrm{D}-\mathrm{N}$ alternating algorithm is devised for the numerical solution of three dimensional Helmholtz problem in an infinite region with an inner spheroid boundary. Firstly, we turn the D-N alternating algorithm into the Richardson iterative algorithm which is equivalent to the original method. Secondly, we prove the convergence of algorithm in the general exterior domain. Then, we give the weak form and discretization of the original equations and prove the convergence of the discretization form. Finally, some numerical examples are presented to illustrate the feasibility and efficiency of this method.

\section{D-N Alternating Algorithm}

Let $(r, \theta, \varphi)$ denote the spherical coordinates; we consider the following 3D exterior Helmholtz problem:

$$
\begin{gathered}
-\Delta u-k^{2} u=f, \quad \text { in } \Omega^{c}, \\
\frac{\partial u}{\partial n}=g, \quad \text { on } \Gamma_{R},
\end{gathered}
$$

where $\Omega$ is a bounded domain in $\mathbb{R}^{3}, \Omega^{c}=\mathbb{R}^{3} \backslash \bar{\Omega}$, and $\Gamma_{R}=$ $\partial \Omega$. $k$ denotes the wave number related to the wavelength $\lambda$ of the incident wave through $k=2 \pi / \lambda$. And assume that $g$ is a known function. In order to assure the existence and uniqueness of the solution of (1), problem (1) satisfies the 
Sommerfeld radiation condition at infinity, which imposes that the scattered wave is outgoing:

$$
\lim _{r \rightarrow \infty} r\left(\frac{\partial u}{\partial r}-i k u\right)=0,
$$

where $\mathrm{i}=\sqrt{-1}, r=|\mathbf{x}|=\sqrt{x^{2}+y^{2}+z^{2}}$. Take a sphere $\Gamma_{1}=\left\{(r, \theta, \varphi) \mid r=R_{1}, 0 \leqslant \theta \leqslant 2 \pi, 0 \leqslant \varphi \leqslant \pi\right\}$ which surrounds $\Gamma_{R}$, such that $\operatorname{dist}\left(\Gamma_{1}, \Gamma_{R}\right)>0$. Then the artificial boundary $\Gamma_{1}$ divides the original unbounded region into two subregions $\Omega_{\text {ext }}$ and $\Omega_{\text {int }}$, where $\Omega_{\text {ext }}$ is the outside subregion and $\Omega_{\text {int }}$ is the bounded subregion surrounded by $\Gamma_{R}$ and $\Gamma_{1}$, $\Omega_{\text {ext }}$ and $\Omega_{\text {int }}$ are nonoverlaping domains. Then the original problem is turned into two subproblems over subdomains $\Omega_{\text {ext }}$ and $\Omega_{\text {int }}$. For exterior boundary value problem (1), we construct the following D-N alternating algorithm:

(i) Step 1. Select the initial $\lambda^{0} \in H^{1 / 2}\left(\Gamma_{1}\right), n=0$.

(ii) Step 2. Solve the Dirichlet problem in $\Omega_{\text {ext }}$ :

$$
\begin{gathered}
-\Delta u_{2}^{n}-k^{2} u_{2}^{n}=0, \quad \text { in } \Omega_{\mathrm{ext}}, \\
u_{2}^{n}=\lambda^{n}, \quad \text { on } \Gamma_{1}, \\
\lim _{r \rightarrow \infty} r\left(\frac{\partial u_{2}^{n}}{\partial r}-\mathrm{i} k u_{2}^{n}\right)=0 .
\end{gathered}
$$

(iii) Step 3. Solve the Neumann problem in $\Omega_{\text {int }}$ :

$$
\begin{gathered}
-\Delta u_{1}^{n}-k^{2} u_{1}^{n}=f, \quad \text { in } \Omega_{\text {int }}, \\
\frac{\partial u_{1}^{n}}{\partial n_{1}}=g, \quad \text { on } \Gamma_{R}, \\
\frac{\partial u_{1}^{n}}{\partial n_{1}}=-\frac{\partial u_{2}^{n}}{\partial n_{2}}, \quad \text { on } \Gamma_{1} .
\end{gathered}
$$

(iv) Step 4. Input $\theta_{n}$, and let

$$
\lambda^{n+1}=\left.\theta_{n} u_{1}^{n}\right|_{\Gamma_{1}}+\left(1-\theta_{n}\right) \lambda^{n}
$$

(v) Step 5. Let $n=n+1$, and go to Step 2,

where $u_{1}^{n}$ and $u_{2}^{n}$ are the $n$th approximate solutions in $\Omega_{\text {int }}$ and $\Omega_{\text {ext }}$, respectively. $n_{1}$ and $n_{2}$ denote the unit outward normals of $\Gamma_{1}$ with respect to two neighboring subdomains; $\theta_{n}$ denotes the $n$th relaxation factor and $\lambda^{0}$ is an arbitrary function in $H^{1 / 2}\left(\Gamma_{1}\right)$. Note that, on interface $\Gamma_{1}$, only the value of the normal derivative of the solution of (3) is needed in solving (4). So it is unnecessary to solve (3). Actually, we can obtain $\partial u_{2}^{n} / \partial n_{2}$ directly from $\lambda^{n}$ by making use of the following natural integral equation [6]:

$$
\frac{\partial u_{2}^{n}}{\partial n_{2}}=\mathscr{K} \lambda^{n}
$$

where $\mathscr{K}$ is the natural integral operator of Helmholtz equation in $\Omega_{\text {ext }}$ :

$$
\frac{\partial u_{2}^{n}}{\partial n_{2}}=-\frac{\partial u_{2}^{n}}{\partial r}(\theta, \varphi)=\sum_{l=0}^{\infty} \sum_{m=-l}^{l} \frac{k h_{l}^{\prime}\left(k R_{1}\right)}{h_{l}\left(k R_{1}\right)} \Lambda_{l}^{m} Y_{l}^{m}(\theta, \varphi),
$$

$h_{l}(\cdot)$ is the first-kind Hankel function of order $l$ and

$$
\begin{gathered}
\Lambda_{l}^{m}=\int_{0}^{2 \pi} \int_{0}^{\pi} \lambda^{n}\left(\theta^{\prime}, \varphi^{\prime}\right) \overline{Y_{l}^{m}\left(\theta^{\prime}, \varphi^{\prime}\right)} \sin \theta^{\prime} \mathrm{d} \theta^{\prime} \mathrm{d} \varphi^{\prime}, \\
Y_{l}^{m}(\theta, \varphi)=\sqrt{\frac{(l-m) !}{(l+m) !} \frac{2 l+1}{4 \pi}} \cdot P_{l}^{m}(\cos \theta) e^{i m \varphi}, \\
\sum_{l=0}^{\infty} \sum_{m=-l}^{l}\left(1+l^{2}\right)^{1 / 2}\left|\Lambda_{l}^{m}\right|^{2}<+\infty,
\end{gathered}
$$

and $P_{l}^{m}(\cdot)$ denotes the associated Legendre function of the first kind, $\overline{Y_{l}^{m}(\cdot)}$ denotes the complex conjugate of $Y_{l}^{m}(\cdot)$.

\section{Equivalent Iterative Method}

To discuss the convergence of the D-N alternating algorithm in Section 2, we first establish a law of deciding. Let $u$ be the exact solution of (1). Denote $\lambda=\gamma_{0} u=\left.u\right|_{\Gamma_{1}}, u_{1}=\left.u\right|_{\Omega_{\text {int }}}$, $u_{2}=\left.u\right|_{\Omega_{\text {ext }}}$; it is easy to know $\lambda \in H^{1 / 2}\left(\Gamma_{1}\right)$, and $u_{1}$ and $u_{2}$ are solutions of the following equations, respectively:

$$
\begin{gathered}
-\Delta u_{1}-k^{2} u_{1}=f, \quad \text { in } \Omega_{\text {int }}, \\
\frac{\partial u_{1}}{\partial n_{1}}=g, \quad \text { on } \Gamma_{R}, \\
u_{1}=\lambda, \quad \text { on } \Gamma_{1}, \\
-\Delta u_{2}-k^{2} u_{2}=0, \quad \text { in } \Omega_{\text {ext }}, \\
u_{2}=\lambda, \quad \text { on } \Gamma_{1}, \\
\lim _{r \rightarrow \infty} r\left(\frac{\partial u_{2}}{\partial r}-\mathrm{i} k u_{2}\right)=0 .
\end{gathered}
$$

By Green formula, we have

$$
\left.\frac{\partial u_{1}}{\partial n_{1}}\right|_{\Gamma_{1}}=-\left.\frac{\partial u_{2}}{\partial n_{2}}\right|_{\Gamma_{1}}
$$

According to superimposition principle, the solutions of (9) and (10) can be denoted

$$
u_{s}=\mathscr{R}_{s} \lambda+\mathscr{T}_{s} f, \quad s=1,2,
$$

where $\mathscr{T}_{s} f(s=1,2)$ satisfy, respectively,

$$
\begin{gathered}
-\Delta \mathscr{T}_{1} f-k^{2} \mathscr{T}_{1} f=f, \quad \text { in } \Omega_{\text {int }}, \\
\frac{\partial \mathscr{T}_{1} f}{\partial n_{1}}=g, \quad \text { on } \Gamma_{R}, \\
\mathscr{T}_{1} f=0, \quad \text { on } \Gamma_{1}, \\
-\Delta \mathscr{T}_{2} f-k^{2} \mathscr{T}_{2} f=0, \quad \text { in } \Omega_{\text {ext }}, \\
\mathscr{T}_{2} f=0, \quad \text { on } \Gamma_{1}, \\
\lim _{r \rightarrow \infty} r\left(\frac{\partial \mathscr{T}_{2} f}{\partial r}-\mathrm{i} k \mathscr{T}_{2} f\right)=0 .
\end{gathered}
$$


It is easy to verify that

$$
\frac{\partial \mathscr{T}_{2} f}{\partial n_{1}}=0
$$

Extend elements of $\Omega_{1}$ and $\Omega_{2}$ by zeros to $\mathscr{R}_{1} \lambda$ and $\mathscr{R}_{2} \lambda$, respectively; therefore $\mathscr{R}_{1} \lambda$ satisfies

$$
\begin{gathered}
-\Delta \mathscr{R}_{1} \lambda-k^{2} \mathscr{R}_{1} \lambda=0, \quad \text { in } \Omega_{\text {int }}, \\
\frac{\partial \mathscr{R}_{1} \lambda}{\partial n_{1}}=0, \quad \text { on } \Gamma_{R}, \\
\mathscr{R}_{1} \lambda=\lambda, \quad \text { on } \Gamma_{1},
\end{gathered}
$$

while $\mathscr{R}_{2} \lambda$ satisfies

$$
\begin{gathered}
-\Delta \mathscr{R}_{2} \lambda-k^{2} \mathscr{R}_{2} \lambda=0, \quad \text { in } \Omega_{\text {ext }}, \\
\mathscr{R}_{2} \lambda=\lambda, \quad \text { on } \Gamma_{1}, \\
\lim _{r \rightarrow \infty} r\left(\frac{\partial \mathscr{R}_{2} \lambda}{\partial r}-\mathrm{i} k \mathscr{R}_{2} \lambda\right)=0,
\end{gathered}
$$

where $\mathscr{R}_{2}$ is the Possion integral operator of Helmholtz equation in the exterior spherical domain $\Omega_{\text {ext }}$. Define

$$
\mathcal{S}_{i}=\frac{\partial \mathscr{R}_{i}}{\partial n_{i}}, \quad i=1,2
$$

$\mathcal{S}_{2}$ is $\mathscr{K}$, which is the natural integral operator of Helmholtz equation in the exterior spherical domain $\Omega_{\text {ext }}$. Substituting (12) in (11), we have

$$
\frac{\partial\left(\mathscr{R}_{1}-\mathscr{R}_{2}\right) \lambda}{\partial n_{1}}=\frac{\partial\left(\mathscr{T}_{2}-\mathscr{T}_{1}\right) f}{\partial n_{1}}=-\frac{\partial \mathscr{T}_{1} f}{\partial n_{1}} .
$$

Namely,

$$
\frac{\partial\left(\mathscr{R}_{1}-\mathscr{R}_{2}\right) \lambda}{\partial n_{1}}=-\frac{\partial \mathscr{T}_{1} f}{\partial n_{1}} .
$$

Define the right-hand term in (19) $\chi=-\partial \mathscr{T}_{1} f / \partial n_{1}$; obviously, $\chi$, a function independent of $\lambda$, can be solved independently by the problem in subdomains in advance. Then, (19) is equivalent to equation

$$
\delta \lambda=\chi
$$

where $\mathcal{S}=\mathcal{S}_{1}+\mathcal{S}_{2}=\mathcal{S}_{1}+\mathscr{K}$ is the Poincaré-Steklov operator [8] on $\Gamma_{1}$. Note that $\lambda \in H^{1 / 2}\left(\Gamma_{1}\right), \mathcal{S}=\mathcal{S}_{1}+\mathcal{S}_{2}=\mathcal{S}_{1}+\mathscr{K}$; it comes that

$$
\mathcal{S}_{1}: H^{1 / 2}\left(\Gamma_{1}\right) \longrightarrow H^{-1 / 2}\left(\Gamma_{1}\right)
$$

Then,

$$
\mathcal{S}: H^{1 / 2}\left(\Gamma_{1}\right) \longrightarrow H^{-1 / 2}\left(\Gamma_{1}\right)
$$

Theorem 1. $\mathcal{S}_{i}: H^{1 / 2}\left(\Gamma_{1}\right) \rightarrow H^{-1 / 2}\left(\Gamma_{1}\right), i=1,2$, is symmetric positive operator.
Proof. We note that $H^{1 / 2}\left(\Gamma_{1}\right) \subset L^{2}\left(\Gamma_{1}\right) \subset H^{-1 / 2}\left(\Gamma_{1}\right)$; therefore, for any $\lambda, \mu \in H^{1 / 2}\left(\Gamma_{1}\right) \subset L^{2}\left(\Gamma_{1}\right)$. By Green formula, we know

$$
\left\langle\mathcal{S}_{i} \lambda, \mu\right\rangle=\int_{\Gamma_{1}} \mathcal{S}_{i} \lambda \mu \mathrm{d} s=\int_{\Gamma_{1}} \frac{\partial \mathscr{R}_{i} \lambda}{\partial n_{i}} \mu \mathrm{d} s=a_{i}\left(\mathscr{R}_{i} \lambda, \mathscr{R}_{i} \mu\right),
$$

where

$$
a_{i}(u, v)=\sum_{s=1}^{3} \int_{\Omega_{\mathrm{ext}}} \frac{\partial u}{\partial x_{s}} \cdot \frac{\partial v}{\partial x_{s}} \mathrm{~d} \mathbf{x}+\int_{\Omega_{\mathrm{ext}}} k^{2} u v \mathrm{~d} \mathbf{x} .
$$

This proves $\mathcal{S}_{i}$ is symmetric positive operator.

Corollary 2. There exists $\mathcal{S}_{i}^{-1}$, which is the inverse operator of $\mathcal{S}_{i}$.

Corollary 3. The bilinear form $a_{i}(\cdot, \cdot)$ on a normed linear space $V_{k}$ is said to be coercive on $H^{1 / 2}\left(\Gamma_{1}\right)$; that is, there exists a positive constant $C$ such that

$$
\left\langle\delta_{k} \lambda, \lambda\right\rangle \geqslant C\|\lambda\|_{H^{1 / 2}\left(\Gamma_{1}\right)}^{2},
$$

where $V_{k}=\left\{v\left|v \in H_{0}^{1}\left(\Omega_{k}\right), v\right|_{\partial \Omega \cap \partial \Omega_{k}}=0, k=1,2,\right\}$.

By Poincaré-Steklov operator, solving the original problem (1) is rewritten as solving the operator equation (20). Generally speaking, it is easier to solve $\mathcal{S}_{1}^{-1}$ than to solve the inverse of $\mathcal{S}$. In fact,

$$
\mathcal{S}_{1} \lambda=h, \quad h \in H^{-1 / 2}\left(\Gamma_{1}\right)
$$

is equivalent to finding $u_{1} \in V_{1}$, such that

$$
a_{1}\left(u_{1}, v\right)=\int_{\Gamma_{1}} h v \mathrm{~d} s, \quad \forall v \in V_{1} .
$$

Let

$$
\lambda=\left.u_{1}\right|_{\Gamma_{1}}
$$

It is easy to verify that

$$
\lambda=\mathcal{S}_{1}^{-1} h \in H^{1 / 2}\left(\Gamma_{1}\right) .
$$

Now we introduce $\mathcal{S}_{1}$ as preprocessor and preconditioned Richardson iterative method. Given $\lambda^{0} \in H^{1 / 2}\left(\Gamma_{1}\right)$, the procedure of iterative

$$
\mathcal{S}_{1}\left(\lambda^{n+1}-\lambda^{n}\right)=\theta_{n}\left(\chi-\mathcal{S} \lambda^{n}\right)
$$

where

$$
\chi=-\frac{\partial}{\partial n_{1}}\left(\mathscr{T}_{1} f\right)
$$

Following [9], to analyze the convergence of algorithm (30), what we need to do is just to estimate the eigenvalues of operator $\mathcal{S}_{1}^{-1} \mathcal{S}$, namely, to estimate the upper bound and the lower bound of $\langle\mathcal{S} \lambda, \lambda\rangle /\left\langle\mathcal{S}_{1} \lambda, \lambda\right\rangle$. 
Lemma 4. There exist two positive constants $\sigma$ and $\tau$, such that

$$
\sigma=\inf _{\lambda \in H^{1 / 2}\left(\Gamma_{1}\right)} \frac{\langle\mathcal{S} \lambda, \lambda\rangle}{\left\langle\mathcal{S}_{1} \lambda, \lambda\right\rangle}, \quad \tau=\sup _{\lambda \in H^{1 / 2}\left(\Gamma_{1}\right)} \frac{\langle\mathcal{S} \lambda, \lambda\rangle}{\left\langle\mathcal{S}_{1} \lambda, \lambda\right\rangle} .
$$

Lemma 5. For any $\lambda \in H^{1 / 2}\left(\Gamma_{1}\right)$, one has $\sigma \leqslant\langle\mathcal{S} \lambda, \lambda\rangle /\left\langle\mathcal{S}_{1} \lambda\right.$, $\lambda\rangle \leqslant \tau$.

Theorem 6. If one puts $\varepsilon=2 / \tau$ and $0<\theta_{n}<\varepsilon$, preconditioned Richardson iterative algorithm (30) is convergent. Particularly, optimal relaxation factor is $\theta_{\text {opt }}=2 /(\sigma+\tau)$. Correspondingly, optimal compression ratio of the iteration is $\delta_{o p t}=(\tau-\sigma) /(\sigma+\tau)$.

Theorem 7. The D-N alternating algorithm (3)-(5) is equivalent to the preconditioned Richardson iterative method (30).

Proof. Let $e_{1}^{n}=u_{1}-u_{1}^{n}$, let $e_{2}^{n}=u_{2}-u_{2}^{n}$, and let $v^{n}=\lambda-\lambda^{n}$. Following (3)-(10), we have

$$
\begin{gathered}
-\Delta e_{2}^{n}-k^{2} e_{2}^{n}=0, \quad \text { in } \Omega_{\mathrm{ext}}, \\
e_{2}^{n}=v^{n}, \quad \text { on } \Gamma_{1}, \\
\lim _{r \rightarrow \infty} r\left(\frac{\partial e_{2}^{n}}{\partial r}-\mathrm{i} k e_{2}^{n}\right)=0, \\
-\Delta e_{1}^{n}-k^{2} e_{1}^{n}=0, \quad \text { on } \Omega_{\mathrm{int}}, \\
e_{1}^{n}=0, \quad \text { on } \Gamma_{R}, \\
\frac{\partial e_{1}^{n}}{\partial n_{1}}=-\frac{\partial e_{2}^{n}}{\partial n_{2}}, \quad \text { on } \Gamma_{1}, \\
v^{n+1}=\left.\theta_{n} e_{1}^{n}\right|_{\Gamma_{1}}+\left(1-\theta_{n}\right) v^{n} .
\end{gathered}
$$

Meanwhile,

$$
e_{2}^{n}=\mathscr{R}_{2} v^{n}, \quad e_{1}^{n}=\mathscr{R}_{1}\left(e_{1}^{n}\right) .
$$

Substituting the above equation to the third equation of (34), we can obtain

$$
\mathcal{S}_{1}\left(e_{1}^{n}\right)=-\mathcal{S}_{2}\left(v^{n}\right)=-\mathscr{K}\left(v^{n}\right) .
$$

Therefore,

$$
\begin{aligned}
& \mathcal{S}_{1}\left(v^{n+1}-v^{n}\right)=\mathcal{S}_{1}\left(\left.\theta_{n} e_{1}^{n}\right|_{\Gamma_{1}}-\theta_{n} v^{n}\right) \\
& =-\theta_{n}\left(\mathscr{K} v^{n}\right)-\theta_{n}\left(\mathcal{S}_{1} v^{n}\right)=-\theta_{n} \delta v^{n} ;
\end{aligned}
$$

thus,

$$
\mathcal{S}_{1}\left(\lambda^{n+1}-\lambda^{n}\right)=\theta_{n} \mathcal{S}\left(\lambda-\lambda^{n}\right)
$$

It comes that

$$
\begin{aligned}
\mathcal{S} \lambda & =\frac{\partial}{\partial n_{1}}\left(\mathscr{R}_{1} \lambda\right)+\frac{\partial}{\partial n_{2}}\left(\mathscr{R}_{2} \lambda\right) \\
& =\frac{\partial}{\partial n_{1}}\left(u_{1}-\mathscr{T}_{1} f\right)+\frac{\partial u_{2}}{\partial n_{2}}=-\frac{\partial \mathscr{T}_{1} f}{\partial n_{1}}=\chi .
\end{aligned}
$$

Substituting the above equation in (39), we have

$$
\mathcal{S}_{1}\left(\lambda^{n+1}-\lambda^{n}\right)=\theta_{n}\left(\chi-\delta \lambda^{n}\right)
$$

\section{Convergence of the Algorithm}

Considering a special case in which $\Gamma_{0}=\{(r, \theta, \varphi) \mid r=$ $\left.R_{0}, 0 \leqslant \theta \leqslant 2 \pi, 0 \leqslant \varphi \leqslant \pi\right\}$ and the interface $\Gamma_{1}=\{(r, \theta, \varphi) \mid$ $\left.r=R_{1}, 0 \leqslant \theta \leqslant 2 \pi, 0 \leqslant \varphi \leqslant \pi\right\}$, following [10], we have the following.

Lemma 8. Suppose

$$
\begin{array}{r}
f_{n}(x)=\frac{n}{2 n+1}+\frac{n+1}{2 n+1} x^{n+1}, \\
x \in[0,1], \quad n \in Z^{+}=\{0,1,2, \ldots\},
\end{array}
$$

and set

$$
U(x)=\sup _{n \in Z^{+}}\left\{f_{n}(x)\right\}, \quad L(x)=\inf _{n \in Z^{+}}\left\{f_{n}(x)\right\} ;
$$

then, we have

$$
\begin{aligned}
& U(x)= \begin{cases}\frac{1}{2}, & 0 \leqslant x \leqslant \frac{1}{2}, \\
x, & \frac{1}{2}<x \leqslant 1,\end{cases} \\
& L(x)= \begin{cases}f_{0}(x), & 0 \leqslant x \leqslant x_{0}, \\
f_{k+1}(x), & x_{k}<x \leqslant x_{k+1},\end{cases}
\end{aligned}
$$

where $x_{k}$ is the only root of equation $f_{k+1}(x)-f_{k}(x)=0$ in interval $(0,1)$ and $x_{k}<x_{k+1}, k$ is a nonnegative integer.

For $\lambda(\theta, \varphi) \in H^{1 / 2}\left(\Gamma_{1}\right)$, in the $L^{2}$-sense, the following expansion holds

$$
\lambda(\theta, \varphi)=\sum_{l=0}^{\infty} \sum_{m=-l}^{l} \Lambda_{l}^{m} Y_{l}^{m}(\theta, \varphi)
$$

where $P_{l}^{m}(x)$ denotes the associated Legendre function of the first kind; $\overline{Y_{l}^{m}(\theta, \varphi)}$ denotes the complex conjugate of $Y_{l}^{m}$. On the one hand,

$$
\left\langle\mathscr{K}^{c} \lambda, \lambda\right\rangle=R_{1} \sum_{l=0}^{\infty} \sum_{m=-l}^{l}(1+l)\left|\Lambda_{l}^{m}\right|^{2}
$$

satisfies

$$
\begin{gathered}
R_{1}\left\{\sum_{l=0}^{\infty} \sum_{m=-l}^{l}\left(1+l^{2}\right)^{1 / 2}\left|\Lambda_{l}^{m}\right|^{2}\right\}^{1 / 2}<+\infty ; \\
\overline{\Lambda_{l}^{m}}=(-1)^{|m|} \Lambda_{l}^{-m},
\end{gathered}
$$

on the other hand, set

$$
\begin{array}{r}
H_{1}^{N} \lambda=\sum_{l=0}^{N} \sum_{m=-l}^{l} \frac{R_{1}^{l+1} \Lambda_{l}^{m}}{R_{1}^{2 l+1}-R_{0}^{2 l+1}}\left(r^{l}-\frac{R_{0}^{2 l+1}}{r^{l+1}}\right) Y_{l}^{m}(\theta, \varphi), \\
R_{0}<r<R_{1} .
\end{array}
$$

Following [11] and (48), we have

$$
\lim _{N \rightarrow \infty}\left\|H_{1} \lambda-H_{1}^{N} \lambda\right\|_{H^{1}\left(\Omega_{1}\right)}=0 .
$$


According to Green formula and orthogonality of $Y_{l}^{m}(\theta, \varphi)$, it comes that

$$
\begin{aligned}
&\left\langle\mathcal{S}_{1} \lambda, \lambda\right\rangle=\int_{\Omega_{1}} \nabla H_{1} \lambda \cdot \nabla H_{1} \lambda \mathrm{d} \mathbf{x} \\
&=\lim _{M \rightarrow \infty, N \rightarrow \infty} \int_{\Omega_{1}} \nabla H_{1}^{M} \lambda \cdot \nabla H_{1}^{M} \lambda \mathrm{d} \mathbf{x} \\
&=\lim _{M \rightarrow \infty, N \rightarrow \infty} \int_{\Gamma_{1}} \frac{\partial H_{1}^{M} \lambda}{\partial r} H_{1}^{N} \lambda \mathrm{d} s \\
&=\lim _{M \rightarrow \infty, N \rightarrow \infty} \sum_{l=0}^{\min \{M, N\}} \sum_{m=-l}^{l} \frac{l R_{1}^{2 l+1}+(l+1) R_{0}^{2 l+1}}{R_{1}^{2 l+1}-R_{0}^{2 l+1}} \\
& \times R_{1} \Lambda_{l}^{m} \cdot \overline{\Lambda_{l}^{m}} .
\end{aligned}
$$

Lemma 9. Suppose

$$
\begin{gathered}
\left\langle\mathcal{S}_{1} \lambda, \lambda\right\rangle=R_{1} \sum_{l=0}^{\infty} \sum_{m=-l}^{l} \frac{l R_{1}^{2 l+1}+(l+1) R_{0}^{2 l+1}}{R_{1}^{2 l+1}-R_{0}^{2 l+1}}\left|\Lambda_{l}^{m}\right|^{2} ; \\
\langle\mathscr{K} \lambda, \lambda\rangle=R_{1} \sum_{l=0}^{\infty} \sum_{m=-l}^{l}(l+1)\left|\Lambda_{l}^{m}\right|^{2},
\end{gathered}
$$

then,

$$
\frac{1}{U\left(R_{0} / R_{1}\right)} \leqslant \frac{\langle\mathcal{S} \lambda, \lambda\rangle}{\left\langle\mathcal{S}_{1} \lambda, \lambda\right\rangle} \leqslant \frac{1}{L\left(R_{0} / R_{1}\right)}
$$

Theorem 10. If relaxation factor $\theta_{n}$ satisfies

$$
0<\theta_{n}<2 L\left(\frac{R_{0}}{R_{1}}\right)
$$

then the D-N alternating algorithm (3)-(5) converges. Particularly, the optimal relaxation factor is

$$
\theta_{\text {opt }}=\frac{2 U\left(R_{0} / R_{1}\right) L\left(R_{0} / R_{1}\right)}{U\left(R_{0} / R_{1}\right)+L\left(R_{0} / R_{1}\right)},
$$

while the optimal compression ratio of the iteration is

$$
\delta_{o p t}=\frac{U\left(R_{0} / R_{1}\right)-L\left(R_{0} / R_{1}\right)}{U\left(R_{0} / R_{1}\right)+L\left(R_{0} / R_{1}\right)} .
$$

Remark 11. We have analyzed the convergence for exterior spherical domain. Following [10], the convergence analysis for the general exterior domain can be extended from above content. Obviously, the optimal relaxation factor and optimal compression ratio of the iteration depend on $R_{1}$ and the geometry of $\Omega^{c}$ in some way, which is confirmed by our numerical results.

\section{The Weak Form and Discretization}

The weak form of problem (4) is Find $u_{1}^{n} \in H_{g}^{1}\left(\Omega_{\text {int }}\right)$, such that

$$
D_{\Omega_{\mathrm{int}}}\left(u_{1}^{n}, v\right)=\int_{\Omega_{\mathrm{int}}} f v \mathrm{~d} \mathbf{x}-\int_{\Gamma_{1}} v \frac{\partial u_{2}^{n}}{\partial n_{2}} \mathrm{~d} s, \quad \forall v \in H_{0}^{1}\left(\Omega_{\mathrm{int}}\right),
$$

where

$$
\begin{aligned}
& D_{\Omega_{\text {int }}}\left(u_{1}^{n}, v\right)=\int_{\Omega_{\text {int }}}\left(\nabla u_{1}^{n} \cdot \nabla v+k^{2} u_{1}^{n} v\right) \mathrm{d} \mathbf{x}, \\
& H_{g}^{1}\left(\Omega_{\text {int }}\right)=\left\{v\left|v \in H^{1}\left(\Omega_{\text {int }}\right), v\right|_{\Gamma_{R}}=g\right\}, \\
& H_{0}^{1}\left(\Omega_{\text {int }}\right)=\left\{v\left|v \in H^{1}\left(\Omega_{\text {int }}\right), v\right|_{\Gamma_{0}}=0\right\} .
\end{aligned}
$$

Obviously, $\left.v\right|_{\Gamma_{1}} \in H^{1 / 2}\left(\Gamma_{1}\right)$. Following (6), the weak form (56) can be rewritten as Find $u_{1}^{n} \in H_{g}^{1}\left(\Omega_{\text {int }}\right)$, such that

$$
\begin{array}{r}
D_{\Omega_{\mathrm{int}}}\left(u_{1}^{n}, v\right)=\int_{\Omega_{\mathrm{int}}} f v \mathrm{~d} \mathbf{x}-\widehat{D}^{c}\left(\lambda^{n}, v\right), \\
\forall v \in H_{0}^{1}\left(\Omega_{\mathrm{int}}\right), \quad i=1,2,
\end{array}
$$

where

$$
\begin{gathered}
\widehat{D}^{c}\left(\lambda^{n}, v\right)=\left\langle\mathscr{K} \lambda^{n}, v\right\rangle=-\sum_{l=0}^{\infty} \sum_{m=-l}^{l} \frac{k R_{l}^{2} h_{l}^{\prime}(k R)}{h_{l}(k R)}\left(\lambda^{n}\right)_{l}^{m} \overline{V_{l}^{m}}, \\
V_{l}^{m}=\int_{0}^{2 \pi} \int_{0}^{\pi} v\left(\theta^{\prime}, \varphi^{\prime}\right) \overline{Y_{l}^{m}\left(\theta^{\prime}, \varphi^{\prime}\right)} \sin \theta^{\prime} \mathrm{d} \theta^{\prime} \mathrm{d} \varphi^{\prime}, \\
\left(\lambda^{n}\right)_{l}^{m}=\int_{0}^{2 \pi} \int_{0}^{\pi} \lambda^{n}\left(\theta^{\prime}, \varphi^{\prime}\right) Y_{l}^{m}\left(\theta^{\prime}, \varphi^{\prime}\right) \sin \theta^{\prime} \mathrm{d} \theta^{\prime} \mathrm{d} \varphi^{\prime} .
\end{gathered}
$$

(58) and (5) are the weak form of D-N method (3)-(5). In the following, we consider the discretization of this weak form. Discretize $\Omega_{\text {int }}$ into a finite number of element domains. Let $S_{h}\left(\Omega_{\text {int }}\right)$ denote the linear subspace of $H_{0}^{1}\left(\Omega_{\text {int }}\right)$ corresponding to this partition. Define $M_{0}, M_{1}$, and $M_{i}$ to be the sets of all nodes belonging to $\Gamma_{R}, \Gamma_{1}$, and $\Omega_{\text {int }}$, respectively. Denote $U_{A}^{n}$ the approximate value and $L_{A}(\mathbf{x})$ a basis function at node $A$. Obviously,

$$
S_{h}\left(\Omega_{\text {int }}\right)=\operatorname{span}\left\{L_{A^{i}}(\mathbf{x}), L_{A^{1}}(\mathbf{x}) \mid A^{i} \in M_{i}, A^{1} \in M_{1}\right\} .
$$

The finite element approximation of $u_{1}^{n}$ can be expressed as

$$
\begin{aligned}
u_{1 h}^{n}= & \sum_{A^{i} \in M_{i}} U_{A^{i}}^{n} L_{A^{i}}(\mathbf{x})+\sum_{A^{1} \in M_{1}} U_{A^{1}}^{n} L_{A^{1}}(\mathbf{x}) \\
& +\sum_{A^{0} \in M_{0}} g\left(\mathbf{x}_{A^{0}}\right) L_{A^{0}}(\mathbf{x}) .
\end{aligned}
$$

By (61) and (5), we can obtain the following discrete D-N alternating algorithm:

$$
\begin{gathered}
\left(\begin{array}{ccc}
Q^{11} & Q^{1 i} & Q^{10} \\
Q^{i 1} & Q^{i i} & Q^{i 0} \\
Q^{01} & Q^{0 i} & Q^{00}
\end{array}\right)\left(\begin{array}{c}
U_{1}^{n} \\
U_{i}^{n} \\
U_{0}^{n}
\end{array}\right)=\left(\begin{array}{c}
-B \Lambda^{n} \\
0 \\
F^{0}
\end{array}\right), \\
\Lambda^{n+1}=\theta_{n} U_{A^{1}}^{n}+\left(1-\theta_{n}\right) \Lambda^{n},
\end{gathered}
$$


where

$$
\begin{gathered}
\Lambda^{n}=\left[\lambda^{n}\left(A^{1}\right)\right]^{T}, \quad U_{s}^{n}=\left[U_{A^{s}}^{n}\right]^{T}, \\
Q^{s t}=\left[D_{\Omega_{\mathrm{int}}}\left(L_{A^{s}}, L_{B^{t}}\right)\right], \\
s, t=0,1, i, \\
Q^{01}=Q^{10}=0, \quad B=\left[b\left(\gamma_{0} N_{A^{1}}, \gamma_{0} N_{B^{1}}\right)\right], \\
F^{0}=\left[\int_{\Gamma_{R}} g N_{B^{0}} \mathrm{~d} s\right]^{T}, \\
A^{s}, B^{s} \in M_{s}, \quad s=0,1, i .
\end{gathered}
$$

\section{Convergence Analysis for the Discretization Form}

In the following, we consider the convergence of the discretization form.

Theorem 12. The discrete D-N alternating algorithms (62) and (63) are equivalent to the following preconditioned Richardson iteration:

$$
S_{h}^{(1)}\left(\Lambda^{n+1}-\Lambda^{n}\right)=\theta_{n}\left(F^{1}-\delta_{h} \Lambda^{n}\right),
$$

where

$$
\begin{gathered}
S_{h}^{(1)}=Q^{11}-Q^{1 i}\left(Q^{i i}-Q^{i 0}\left(Q^{00}\right)^{-1} Q^{0 i}\right)^{-1} Q^{i 1}, \\
S_{h}=S_{h}^{(1)}+B \\
F^{1}=Q^{1 i}\left(Q^{i i}-Q^{i 0}\left(Q^{00}\right)^{-1} Q^{0 i}\right)^{-1} Q^{i 0}\left(Q^{00}\right)^{-1} F^{0} .
\end{gathered}
$$

Proof. Let $\left[U_{1}, U_{i}, U_{0}\right]^{T}$ be the solution of the equation

$$
\left(\begin{array}{ccc}
Q^{11}+B & Q^{1 i} & Q^{10} \\
Q^{i 1} & Q^{i i} & Q^{i 0} \\
Q^{01} & Q^{0 i} & Q^{00}
\end{array}\right)\left(\begin{array}{c}
U_{1} \\
U_{i} \\
U_{0}
\end{array}\right)=\left(\begin{array}{c}
0 \\
0 \\
F^{0}
\end{array}\right)
$$

Simplified to

$$
\begin{gathered}
{\left[Q^{11}-Q^{1 i}\left(Q^{i i}-Q^{i 0}\left(Q^{00}\right)^{-1} Q^{0 i}\right)^{-1} Q^{i 1}+B\right] U_{1}} \\
=Q^{1 i}\left(Q^{i i}-Q^{i 0}\left(Q^{00}\right)^{-1} Q^{0 i}\right)^{-1} Q^{i 0}\left(Q^{00}\right)^{-1} F^{0},
\end{gathered}
$$

namely,

$$
S_{h} U_{1}=F^{1} .
$$

Then, (67) can be rewritten as

$$
\left(\begin{array}{lll}
Q^{11} & Q^{1 i} & Q^{10} \\
Q^{i 1} & Q^{i i} & Q^{i 0} \\
Q^{01} & Q^{0 i} & Q^{00}
\end{array}\right)\left(\begin{array}{c}
U_{1} \\
U_{i} \\
U_{0}
\end{array}\right)=\left(\begin{array}{c}
-B U_{1} \\
0 \\
F^{0}
\end{array}\right) .
$$

By (62) and (70),

$$
\left(\begin{array}{lll}
Q^{11} & Q^{1 i} & Q^{10} \\
Q^{i 1} & Q^{i i} & Q^{i 0} \\
Q^{01} & Q^{0 i} & Q^{00}
\end{array}\right)\left(\begin{array}{c}
U_{1}^{n}-U_{1} \\
U_{i}^{n}-U_{i} \\
U_{0}^{n}-U_{0}
\end{array}\right)=\left(\begin{array}{c}
-B\left(\Lambda^{n}-U_{1}\right) \\
0 \\
0
\end{array}\right) .
$$

We have

$$
S_{h}^{(1)}\left(U_{1}^{n}-U_{1}\right)=B\left(U_{1}-\Lambda^{n}\right) .
$$

Therefore,

$$
\begin{aligned}
S_{h}^{(1)} & \left(\Lambda^{n+1}-\Lambda^{n}\right)=\theta_{n} S_{h}^{(1)}\left(U_{1}^{n}-\Lambda^{n}\right) \\
& =\theta_{n}\left[S_{h}^{(1)}\left(U_{1}^{n}-U_{1}\right)+S_{h}^{(1)}\left(U_{1}-\Lambda^{n}\right)\right] \\
& =\theta_{n}\left(B+S_{h}^{(1)}\right)\left(U_{1}-\Lambda^{n}\right) \\
& =\theta_{n} S_{h}\left(U_{1}-\Lambda^{n}\right) \\
& =\theta_{n}\left(F^{1}-S_{h} \Lambda^{n}\right) .
\end{aligned}
$$

Theorem 13. Let $\alpha$ be spectral radius of $\left(S_{h}^{(1)}\right)^{-1} S_{h}$, which is iterative matrix of preconditioned Richardson iteration. Then, there is a positive constant $\beta$, which is independent of finite element mesh parameter $h$ of subdomain $\Omega_{\mathrm{int}}$, such that $\alpha \leqslant \beta$.

Theorem 14. Eigenvalues of iterative matrix $\left(S_{h}^{(1)}\right)^{-1} S_{h}$ are real and greater than 1 .

Theorem 15. Put $\theta_{n}=\eta(n=0,1, \ldots)$; then, there exists a constant $\delta(0<\delta<1)$, which is independent of finite element mesh parameter $h$ of subdomain $\Omega_{\text {int }}$. For $0<$ $\eta<\delta$, the preconditioned Richardson iteration converges, the discrete $D-N$ alternating algorithm (62)-(63) converges and the convergence rate is independent of mesh parameter $h$ of subdomain $\Omega_{\text {int }}$.

Proof. By (73), we have

$$
\begin{aligned}
U_{1}-\Lambda^{n+1} & =\left[I-\eta\left(S_{h}^{(1)}\right)^{-1} S_{h}\right]\left(U_{1}-\Lambda^{n}\right) \\
& =\left[I-\eta\left(S_{h}^{(1)}\right)^{-1} S_{h}\right]^{n+1}\left(U_{1}-\Lambda^{0}\right) ;
\end{aligned}
$$

it comes that

$$
\left\|U_{1}-\Lambda^{n+1}\right\|_{2} \leqslant \delta^{n+1}\left\|U_{1}-\Lambda^{0}\right\|_{2}
$$

where

$$
\delta=\left\|I-\eta\left(S_{h}^{(1)}\right)^{-1} S_{h}\right\|_{2} .
$$

Following Theorem 13 and Theorem 14, there exists a constant $\delta(0<\delta<1)$, which is independent of $h$. For $0<\eta<\sigma$, spectral radius of $I-\eta\left(S_{h}^{(1)}\right)^{-1} S_{h}$ is less than 1 , and spectral norm $\delta<1$; therefore,

$$
\lim _{n \rightarrow+\infty}\left\|U_{1}-\Lambda^{n+1}\right\|_{2}=0 .
$$

It follows that the preconditioned Richardson iteration converges; then, the discrete $\mathrm{D}-\mathrm{N}$ alternating algorithm (62)-(63) 
TABLE 1: Maximum node-error on $\bar{\Omega}_{i}$ for $R_{1}=2.0$.

\begin{tabular}{ccccccc}
\hline$\theta$ & \multicolumn{7}{c}{ Iterative times } \\
& 0 & 1 & 2 & 3 & 4 & 12 \\
\hline 0.3 & 0.33062 & 0.18917 & 0.11558 & 0.07835 & 0.05963 & 0.04858 \\
0.4 & 0.33062 & 0.14107 & 0.07438 & 0.05198 & 0.04806 & 0.04713 \\
0.5 & 0.33062 & 0.08362 & 0.05926 & 0.04816 & 0.04823 & 0.04818 \\
0.6 & 0.33062 & 0.06345 & 0.09187 & 0.06537 & 0.06823 & 0.09684 \\
0.7 & 0.33062 & 0.11998 & 0.15164 & 0.06356 & 0.08451 & 0.06529 \\
\hline
\end{tabular}

TABLE 2: Maximum node-error on $\bar{\Omega}_{i}$ for $R_{1}=6.0$.

\begin{tabular}{ccccccc}
\hline$\theta_{n}$ & \multicolumn{7}{c}{ Iterative times } \\
& 0 & 1 & 2 & 3 & 4 & 12 \\
\hline 0.3 & 0.13381 & 0.05442 & 0.04968 & 0.04930 & 0.04927 & 0.04927 \\
0.4 & 0.13381 & 0.03435 & 0.05269 & 0.04849 & 0.49448 & 0.04927 \\
0.5 & 0.13381 & 0.04332 & 0.06801 & 0.03922 & 0.05465 & 0.04939 \\
0.6 & 0.13381 & 0.11119 & 0.14317 & 0.07156 & 0.11630 & 0.08039 \\
0.7 & 0.13381 & 0.16126 & 0.24338 & 0.22273 & 0.31370 & 0.63435 \\
\hline
\end{tabular}

converges and the convergence rate is independent of mesh parameter $h$ of subdomain $\Omega_{\text {int }}$.

\section{Numerical Examples}

To test the effectiveness of the method in this paper, we give two numerical examples, using the discrete D-N alternating algorithm in Section 2. In the two examples, the exact solutions are known. The purpose of these examples is to check the convergence in terms of iteration and mesh size $h$.

Suppose that $\Omega^{c}=\{(x, y, z)|| x|>1| y,|>1| z \mid,>1\}$ is the exterior domain of cube $[-1,1] \times[-1,1] \times[-1,1]$. The artificial boundary is $\Gamma_{1}=\left\{(r, \theta, \varphi) \mid r=R_{1}, R_{1}>\right.$ $\sqrt{3}\}, r=\sqrt{x^{2}+y^{2}+z^{2}}$. Split the bounded domain $\Omega_{\text {int }}$ into finite element mesh as follows. Firstly, make every inner boundary edge into $N_{1}$ equivalent parts and connect nodes by using segments which are parallel to the corresponding coordinate axis; then, the grid located in the inner boundary $\Gamma_{1}$ is generated. Secondly, along the radial direction, rays starting from each node of located in the inner boundary intersect artificial boundaries at some nodes, and all the other nodes are produced by dividing segments along the radial direction into $\mathrm{N}_{2}$ equivalent parts. At last, we get an eightnode trilinear isoparametric finite elements of $\Omega_{\text {int }}$.

Denote $e$ the maximum node-error on $\bar{\Omega}_{i}$ :

$$
e(n)=\sup _{P_{i} \in \bar{\Omega}_{i}}\left|u\left(P_{i}\right)-u_{1 h}^{n}\left(P_{i}\right)\right|
$$

$e_{h}$ denotes the maximum node-error of the abjacent twosteps on nodes

$$
e_{h}(n)=\sup _{P_{i} \in \bar{\Omega}_{i}}\left|u_{1 h}^{n+1}\left(P_{i}\right)-u_{1 h}^{n}\left(P_{i}\right)\right|
$$

TABLE 3: The relation between convergence rate and relaxation factor.

\begin{tabular}{cccccccc}
\hline & $\theta_{n}$ & \multicolumn{6}{c}{ Iterative times } \\
& & 0 & 1 & 2 & 3 & 4 & 12 \\
\hline \multirow{4}{*}{0.2} & $e$ & 0.13381 & 0.07449 & 0.05900 & 0.05302 & 0.05072 & 0.04928 \\
& $e_{h}$ & & 0.02358 & 0.05223 & 0.01157 & 0.00256 & 0.00057 \\
& $q_{h}$ & & & 4.51432 & 4.51329 & 4.51206 & 4.51101 \\
\hline \multirow{4}{*}{0.3} & $e$ & 0.13381 & 0.54416 & 0.04968 & 0.04330 & 0.04927 & 0.04927 \\
& $e_{h}$ & 0 & 0.50323 & 0.0806 & 0.01294 & 0.00209 & 0.00031 \\
& $q_{h}$ & 0 & 0 & 6.2432 & 6.2301 & 6.1952 & 6.1809 \\
\hline \multirow{4}{*}{0.4} & $e$ & 0.13381 & 0.03434 & 0.05268 & 0.04849 & 0.04945 & 0.04927 \\
& $e_{h}$ & 0 & 0.01512 & 0.00176 & 0.00021 & 0.00002 & 0 \\
& $q_{h}$ & 0 & 0 & 8.5961 & 8.5532 & 8.3201 & 7.9326 \\
\hline
\end{tabular}

$q_{h}$ is the convergence rate

$$
q_{h}(n)=\frac{e_{h}(n-1)}{e_{h}(n)} .
$$

We substitute $\sum_{l=0}^{M}$ for $\sum_{l=0}^{\infty}$ in the computing of the entries of $K_{h}$. Denote by $m$ the total number of nodes on $\overline{\Omega_{\text {int }}}$. By computing, the results are as follows.

Example 16. In the problem (1), we take

$$
g=\sqrt{\frac{3}{4 \pi}}\left(\frac{k^{2} \mathrm{i}-2 \mathrm{i}-2 k}{k^{2}}\right) e^{\mathrm{i} k} \cos \theta ;
$$

the exact solution is

$$
u(r, \theta)=-\sqrt{\frac{3}{4 \pi}} \frac{k r+\mathrm{i}}{k^{2} r^{2}} e^{\mathrm{i} k r} \cos \theta .
$$

Let $k=2.0, N_{1}=N_{2}=2, m=78, M=4$, the numerical results are shown in Tables 1 and 2.

Tables 1 and 2 show that the D-N alternating algorithm (62)-(63) is convergent. Table 1 shows that the speed of convergence is faster while $\theta_{n}=0.5$. Conversely, Table 2 shows the different phenomenon that the speed of convergence is faster while $\theta_{n}=0.3$. It proves Theorem 10 and shows relaxation factor $\theta_{n}$ is dependent on $R_{1}$. But it is valuable to know that $\theta_{n}=0.5$ unsatisfied the requirement of Theorem $10\left(2 L\left(R_{0} / R_{1}\right)=0.5\right)$, but the $\mathrm{D}-\mathrm{N}$ alternating algorithm (62)-(63) is convergent, which shows (53) is the sufficient condition of Theorem 10.

Example 17. In the problem (1), we take

$$
g=\sqrt{\frac{15}{32 \pi}}\left(\frac{k^{3}+4 k^{2} \mathrm{i}-9 k-9 \mathrm{i}}{k^{3}}\right) e^{\mathrm{i}(k+\phi)} \sin 2 \theta ;
$$

the exact solution is

$$
u(r, \theta)=-\sqrt{\frac{15}{32 \pi}}\left(\frac{3 \mathrm{i}}{k^{3} r^{3}}+\frac{3}{k^{2} r^{2}}-\frac{\mathrm{i}}{k r}\right) e^{\mathrm{i}(k r+\varphi)} \sin 2 \theta .
$$

Let $k=2.0, R_{1}=6.0, N_{1}=N_{2}=2, m=78, M=4$, the numerical results are shown in Table 3 . 
TABLE 4: The relation between convergence rate and mesh.

\begin{tabular}{cccccccc}
\hline & Mesh & \multicolumn{6}{c}{ Iterative times } \\
& & 0 & 1 & 2 & 3 & 4 & 12 \\
\hline \multirow{3}{*}{$\mathrm{I}$} & $e$ & 0.16651 & 0.12025 & 0.11342 & 0.11354 & 0.11339 & 0.11327 \\
& $e_{h}$ & & 0.11547 & 0.10300 & 0.08651 & 0.07381 & 0.06525 \\
& $q_{h}$ & & & 1.12105 & 1.19058 & 1.17205 & 1.13114 \\
\hline \multirow{3}{*}{$\mathrm{II}$} & $e$ & 0.13381 & 0.03434 & 0.05268 & 0.04849 & 0.04945 & 0.04927 \\
& $e_{h}$ & & 0.02834 & 0.01814 & 0.01162 & 0.00745 & 0.00478 \\
& $q_{h}$ & & & 1.56223 & 1.56134 & 1.56001 & 1.56018 \\
\hline \multirow{3}{*}{ III } & $e$ & 0.08874 & 0.01239 & 0.01753 & 0.01623 & 0.01648 & 0.01644 \\
& $e_{h}$ & & 0.01056 & 0.00449 & 0.00191 & 0.00083 & 0.00036 \\
& $q_{h}$ & & & 2.35073 & 2.35327 & 2.30124 & 2.29801 \\
\hline
\end{tabular}

Table 3 shows that in the scope of relaxation factor, the bigger the relaxation factor $\theta_{n}$ is, the faster the convergence rate is.

While we put $\theta_{n}=0.4$ and $R_{1}=6.0$ and denote Mesh I: $N_{1}=N_{2}=1, m=16, M=4$; Mesh II: $N_{1}=N_{2}=2$, $m=78, M=4$; Mesh III: $N_{1}=N_{2}=4, m=490, M=4$. The numerical results are shown in Table 4.

Table 4 shows that the convergence rate of the discrete D$\mathrm{N}$ alternating algorithm is independent of mesh parameter $h$. At the same time, the closer we refine the mesh, the smaller the maximum node-error will be. The maximum node-error is roughly of $\mathrm{O}(h)$ order.

\section{Conflict of Interests}

The authors declare that there is no conflict of interests regarding the publication of this paper.

\section{Acknowledgments}

The research is supported by the National Natural Science Foundantion of China, contact/Grant no. 11371198, Jiangsu Provincial Natural Science Foundation of China, contact/Grant no. BK20141008, and the Jiangsu Provincial Key Laboratory for Numerical Simulation of Large Scale Complex Systems contract/Grant no. 201305.

\section{References}

[1] D. Givoli, Numerical Methods for Problems in Infinite Domains, Elsevier, Amsterdam, The Netherlands, 1992.

[2] I. Harari and T. J. R. Hughes, "Analysis of continuous formulations underlying the computation of time-harmonic acoustics in exterior domains," Computer Methods in Applied Mechanics and Engineering, vol. 97, no. 1, pp. 103-124, 1992.

[3] D. Givoli and J. B. Keller, "A finite element method for large domains," Computer Methods in Applied Mechanics and Engineering, vol. 76, no. 1, pp. 41-66, 1989.

[4] D. H. Yu, "Discretization of non-overlapping domain decomposition method for un-bounded domains and its covergence," Mathematica Numerica Sinica, vol. 18, no. 3, pp. 328-336, 1996.

[5] D. H. Yu and Z. P. Jia, "The nonoverlapping domain decomposition method based on natural boundary reduction for a 2-dimensional exterior Helmholtz problem," Mathematica Numerica Sinica. Jisuan Shuxue, vol. 22, no. 2, pp. 227-240, 2000.

[6] J. M. Wu and D. H. Yu, "The natural integral equations of 3-D exterior Helmholtz problem and its numerical solution," Chinese Journal of Computational Physics, vol. 16, no. 5, pp. 449456, 1999.

[7] Z. P. Jia, J. M. Wu, and D. H. Yu, "A coupled natural boundary element and finite element method for solving a 3-dimensional exterior Helmholtz problem," Mathematica Numerica Sinica, vol. 23, no. 3, pp. 357-368, 2001.

[8] J. C. Xu and S. Zhang, "Preconditioning the Poincaré-Steklov operator by using Green's function," Mathematics of Computation, vol. 66, no. 217, pp. 125-138, 1997.

[9] T. Läu, J. M. Shi, and Z. B. Lin, Domain Decomposition Method. A New Technique for Numereical Solution of Partial Differential Equations, Science Press, Beijing, China, 1992.

[10] D. Yu and J. M. Wu, "A nonoverlapping domain decomposition method for exterior 3-D problem," Journal of Computational Mathematics, vol. 19, no. 1, pp. 77-86, 2001.

[11] J. M. Wu and D. H. Yu, "The natural integral equations of 3-D harmonic problems and their numerical solutions," Mathematica Numerica Sinica, vol. 20, no. 4, pp. 419-430, 1998. 


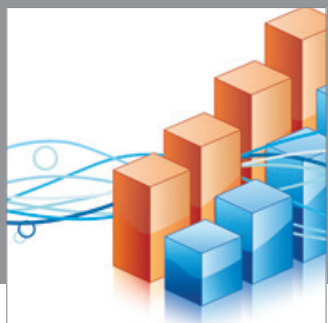

Advances in

Operations Research

mansans

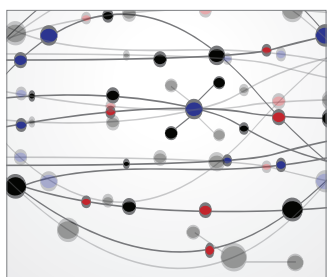

The Scientific World Journal
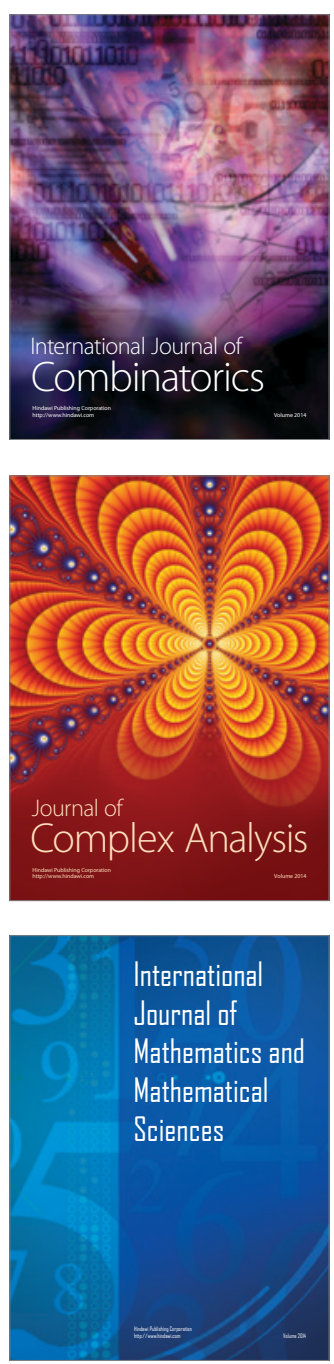
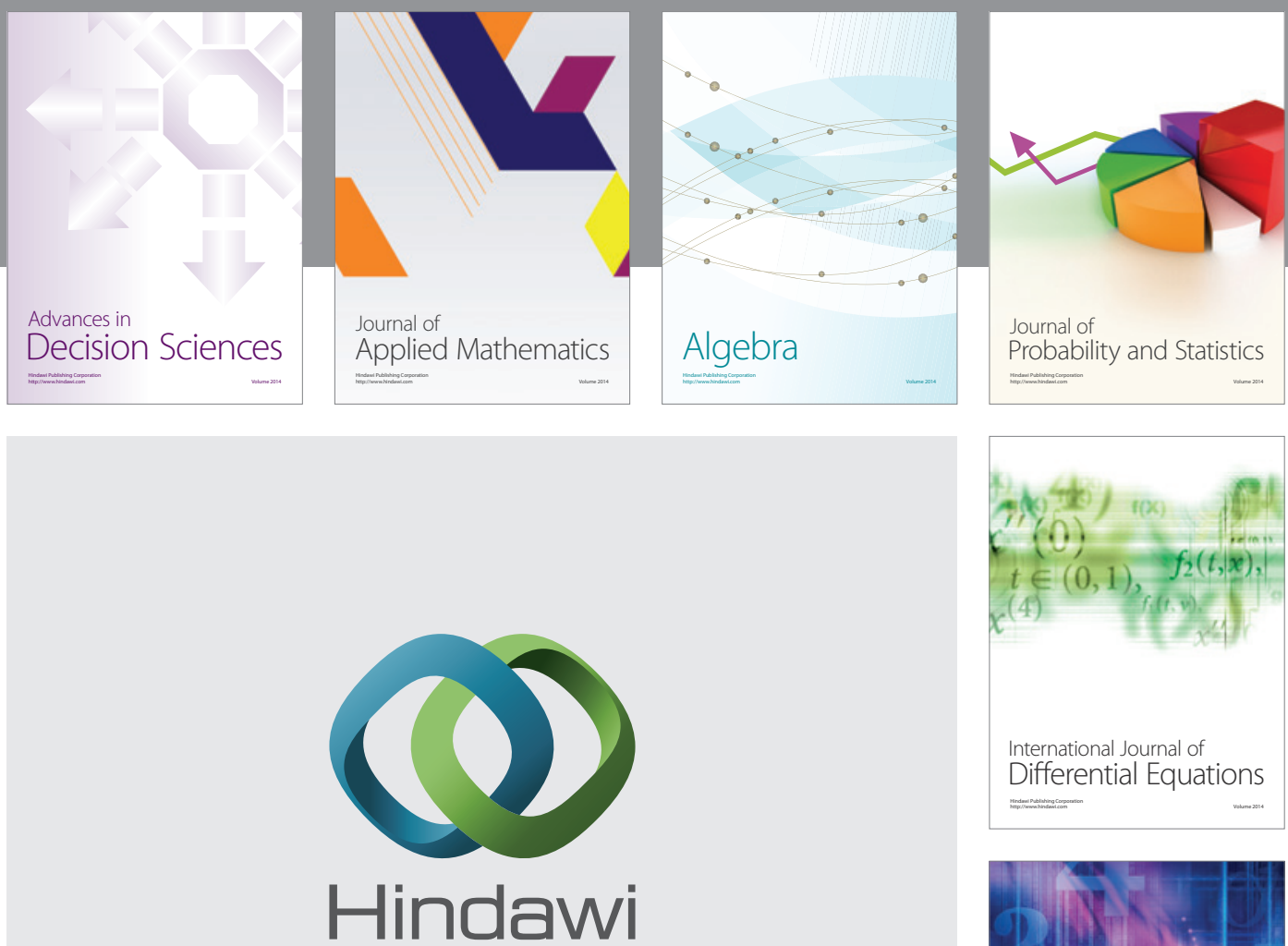

Submit your manuscripts at http://www.hindawi.com
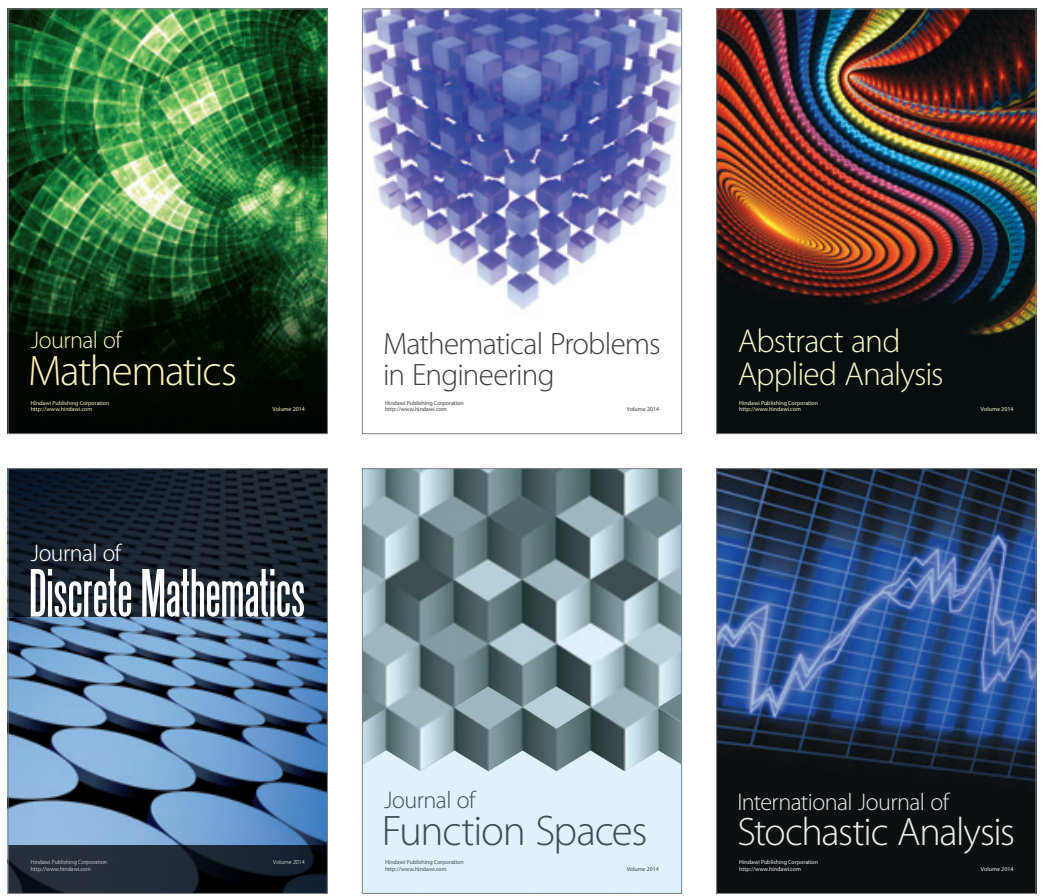

Journal of

Function Spaces

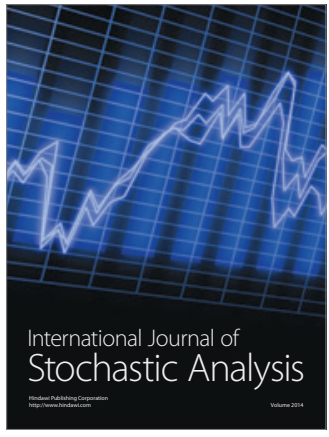

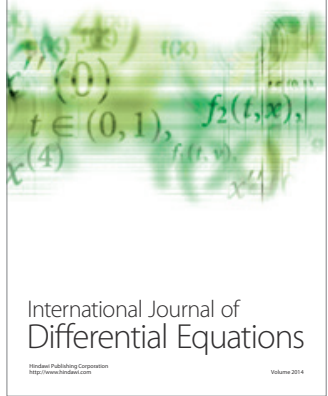
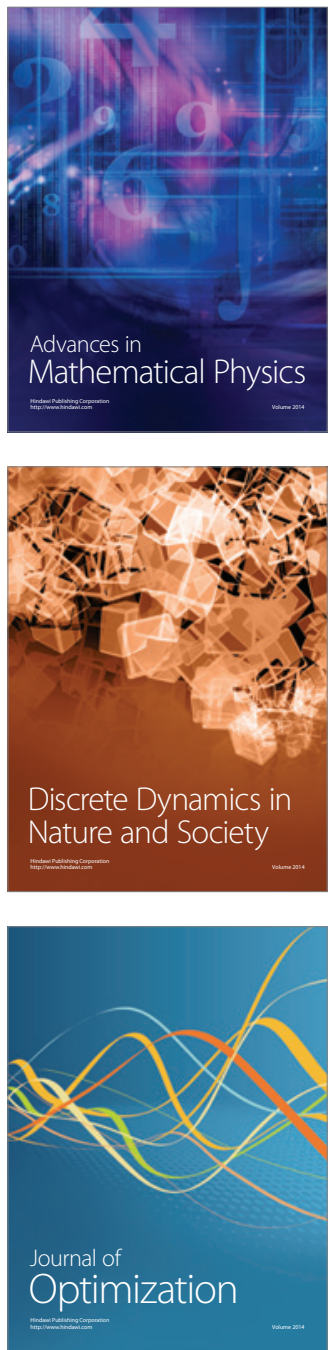